\title{
Surveiller pour mieux voter. Les élections municipales de l'été 1848 dans le département du Finistère
}

\section{Laurent Le Gall}

\section{(2) OpenEdition Journals}

Édition électronique

URL : http://journals.openedition.org/conflits/993

DOI : $10.4000 /$ conflits.993

ISSN : 1777-5345

Éditeur :

CCLS - Centre d'études sur les conflits lilberté et sécurité, L'Harmattan

Édition imprimée

Date de publication : 1 mars 2004

Pagination : 43-69

ISBN : 2-7475-6791-5

ISSN : 1157-996X

Référence électronique

Laurent Le Gall, « Surveiller pour mieux voter. Les élections municipales de l'été 1848 dans le département du Finistère », Cultures \& Conflits [En ligne], 53 I printemps 2004, mis en ligne le 04 octobre 2004, consulté le 30 mars 2021. URL : http://journals.openedition.org/conflits/993 ; DOI : https://doi.org/10.4000/conflits.993

Ce document a été généré automatiquement le 30 mars 2021

Creative Commons License 


\title{
Surveiller pour mieux voter. Les élections municipales de l'été 1848 dans le département du Finistère
}

\author{
Laurent Le Gall
}

Surveillance politique et élections au XIXème siècle : le lien semble aller de soi, tant la version progressiste de l'émancipation démocratique qui imprègne nombre d'études consacrées à la vie politique et à une de ses manifestations les plus tangibles, le vote, ne cesse de rappeler combien l'autonomie du sujet politique s'est aussi construite contre l'étau d'un ordre étatico-politique ${ }^{1}$ soucieux de peser sur le choix et le destin de la représentation nationale. Moment sensible, le moment électoral, au fur et à mesure qu'il s'installait comme un pic récurrent du temps politique, s'est vu investi d'enjeux opposés et paradoxaux en apparence: encadré par des normes de plus en plus contraignantes et impératives, surveillé étroitement afin d'éviter le débordement ou la déstabilisation, le scrutin s'est parfois transformé en une formidable caisse de résonance pour les acteurs-contemplateurs du champ politique ${ }^{2}$, en un théatre des fièvres passagères. Comme si la surveillance sécrétait, chez certains, une possible mise en scène de leur individualité politique. Ainsi, l'apprentissage de la civilité électorale, les processus de «disciplinarisation » et de domestication de l'électeur ${ }^{3}$, l'acceptation d'un savoir-faire et d'un savoir-être au moment du vote, ont eu partie liée avec un arsenal législatif, judiciaire et parfois répressif, progressivement adapté aux nouvelles contingences électorales. Surveiller pour mieux voter, tel peut être, en résumé, la façon avec laquelle des agents de l'Etat ont pu imaginer la fabrique d'un ordre démocratique strictement et fermement délimité. Instrument de régulation du social par le politique, l'élection a imposé une surveillance promue par des autorités administratives garantes de la sécurité des participants, de la légalité du scrutin, de la légitimité des résultats. Une surveillance imposée qui a pu induire, en retour, un raidissement des enjeux électoraux, une tendance à la conflictuosité, et pour certains, le plaisir du contournement de la norme et celui de la dérobade.

Surveillance politique et élections sont donc intimement liées, dans un jeu de va-etvient qui permet d'inscrire le mode de dévolution du pouvoir dans un horizon d'étude 
faisant la part belle aux interactions entre normes prescrites et normes vécues, fabrication des mécanismes de l'Etat et intériorisation - partielle ou totale, selon des modes d'acceptation ou de refus différents - de ces derniers, tant au niveau d'un groupe qu'au niveau de l'individu. Avec un bémol cependant. Car le tableau qui vient d'être succinctement brossé d'une surveillance politique visible lors des élections générales vaut-il pour des scrutins locaux frappés, aux yeux de la plupart des élites, d'une inconsistance politique chronique? C'est ce que cette étude, qui espère dépasser le cadre strict de la monographie locale, se propose de comprendre à partir de l'exemple des scrutins municipaux de l'été 1848 dans le Finistère; alors que le suffrage devenu universel transformait possiblement, comme partout ailleurs, l'horizon politique de millions d'électeurs.

Les élections municipales : l'oeil du pouvoir absent?

Les élections municipales seraient-elles des élections sous surveillance politique pour des élections sans politique? Dans la rhétorique administrative, le scrutin des 30 et 31 juillet 1848 ne tranche pas avec les élections locales précédentes, instituées dans certaines communes depuis 1831 : c'est une opération sans enjeux politiques, marquée au sceau des querelles de village, des susceptibilités de notables communaux, des haines de faction. Pour les élites politico-administratives, il ne faisait donc aucun doute que le scrutin au suffrage universel obéirait aux mêmes logiques d'une conquête du pouvoir liées fondamentalement à des intérêts particuliers qui ne pouvaient qu'échapper aux observateurs les plus avisés - sous-préfets, juges de paix, gendarmes, par exemple - de ces micro-sociétés organisées à l'ombre du pouvoir central. Dans les journaux, quelques entrefilets mentionnent le retour, communal cette fois-ci, vers les urnes. Quant au préfet, il se borne à faire insérer dans le bulletin administratif, l'arrêté de convocation des assemblées électorales et les informations relatives au décret du 3 juillet 1848, et à rappeler, au passage, les modalités concernant le maintien de l'ordre au moment des élections : conformément à la loi du 21 mars 1831, «le président a seul la police de l'assemblée (art. 48) et le bureau (art. 50) juge provisoirement les difficultés qui s'élèvent sur les opérations $»^{4}$. Sans enjeux nationaux, ne risquant pas de mettre en péril le devenir de la République, ces élections en mineur, cantonnées aux 282 microcosmes communaux, n'incitaient donc pas les autorités administratives à les entourer d'une surveillance politique particulière.

On était loin des mesures préventives, préconisées en avril 1848 dans un climat d'effervescence, d'attente et de suspicion, pour proscrire tout incident et assurer le bon déroulement des élections à l'Assemblée constituante ${ }^{5}$. Conformément aux instructions du commissaire de la République qui avait intimé l'ordre aux maires de faire respecter la liberté du vote, il était noté, par exemple, dans le procès-verbal du canton de Lesneven : "A la réquisition du président, pour la police de l'assemblée et pour faciliter l'entrée et la sortie de la salle[la mairie],des factionnaires ont été placés aux deux portes ${ }^{6}$. A PontAven, le scrutin était clos le 25 avril en tout début d'après-midi et le formulaire de réception des suffrages précisait : «A deux heures et quart le bureau étant au complet(...) le Président a déclaré qu'il allait procéder au compte des votes que renferme la boîte du scrutin. En conséquence, tous les bulletins ayant été retirés de la boîte électorale et comptés en présence du public, qui a vu en faire vingt-quatre paquets scellés, renfermant, sauf erreur, cent chacun, et un vingt-cinquième paquet, qui ne refermait que quarante-trois, aussi sauf erreur. Tous ces paquets ont été,au vu et au su de tous les électeurs présents, qui étaient en grand nombre, déposés dans la boîte électorale, qui, comme les précédents jours, a été scellée, cachetée, et déposée dans l'armoire de la salle de la mairie, et une nouvelle bande de scellé y a été apposée en présence du 
bureau entier et du public; et la garde de ce dépôt précieux a été confiée à douze gardes nationaux, qui seront accompagnés d'un gendarme de cette résidence (...) $\|^{8}$. D'autres procèsverbaux mentionnent la présence de forces chargées de prévenir le tumulte et l'incident, même si beaucoup ne sont pas aussi explicites. Retenons ici deux éléments. En premier lieu, le scrutin est un acte éminemment public puisqu'il engage l'honneur d'une communauté de citoyens à la fois électeurs et votants, mais aussi observateurs de la bonne organisation de ces élections. Ensuite, le bon déroulement du scrutin est fréquemment conditionné à la présence d'une force de maintien de l'ordre qui encadre ce moment électoral, au nom d'une morale de l'Etat dont sont dépositaires, en plus du bureau électoral et du maire, gendarmes, gardes champêtres et gardes nationaux : des hommes qui surveillent et maintiennent l'ordre ponctuellement ou au nom de leur fonction sociale ; des hommes qui se situaient à l'intérieur de la communauté - par leur mode de vie, leur intégration économique et familiale - mais s'en écartaient aussi puisqu'ils étaient un des maillons, même s'ils étaient en bout de chaîne, d'un contrôle étatique, et ici politique, de plus en plus sourcilleux et prégnant ${ }^{9}$.

Les élections municipales de l'été 1848 apparaissent donc comme des élections sans surveillance politique prescrite par les agents de l'Etat. Comme si elles ne s'intégraient que dans un système de dévolution du pouvoir obéissant certes à des règles nationales mais foncièrement inféodé à des pratiques communales endogènes. Rares sont, en effet, les procès-verbaux qui mentionnent l'existence d'une force publique supplétive chargée de contenir les désordres possibles et de contrôler le bon déroulement des opérations. Dans la très grande commune de Kerlouan (plus de 3500 habitants, 764 électeurs répartis en 3 sections, 459 votants), le scrutin est clos, sur le procès-verbal, ce 30 juillet à 11 heures. Une protestation datée du 6 août détaille le moment qui précéda le dépouillement: "A onze heures et demie, on s'est réuni à la maison commune où les urnes ont été déposées en présence des électeurs assemblés. D'un commun accord entre les membres des bureaux et les électeurs, il a été convenu de suspendre la séance pendant une heure avant de commencer le dépouillement. Pendant cette suspension, les urnes déposées à la mairie y sont restées sous la garde de deux factionnaires qui se tenaient en dehors de la porte fermée et la clef est restée entre les mains du citoyen maire $»^{10}$. A Crozon, un gendarme est présent pendant la durée des opérations ${ }^{11}$. Il est évident que le possible recours à une force chargée du maintien de l'ordre dépendait de nombreux facteurs: la présence d'une brigade de gendarmerie, l'existence d'un garde champêtre rétribué, l'état et l'efficacité d'une garde nationale locale souvent fantomatique et renaissant le jour de son élection, les signes avant-coureurs des tensions et des perturbations attendues au moment du scrutin, la volonté de se conformer, par mimétisme, aux procédures de surveillance mises en place au printemps.

Elections en mineur pour les élites administratives, ces élections qui, d'après leur version de la politique, ne risquaient pas d'importer la République au village, ont pourtant fait couler beaucoup d'encre. Il n'est pas dans notre propos de nous intéresser à l'essence du pouvoir au village au début de la Seconde République, et de discuter, par exemple, la question de «la démocratie impossible» - au cours d'une période qui magnifie la démocratie républicaine - dans les communes rurales ${ }^{12}$. Esquissons seulement ce qui fit la spécificité des scrutins municipaux de l'été 1848 qui perpétuaient la geste électorale locale entamée une quinzaine d'années plus tôt, et dont Christine Guionnet a analysé les linéaments et les modalités ${ }^{13}$ : avec le suffrage universel, le champ politique local se dilate et se diffracte aussi, dans la mesure où l'universalité du suffrage aiguise bien des appétits; l'opération des 30 et 31 juillet 
suppose pour certains votants la réitération d'un acte qu'ils ont accompli, en trois mois, à deux autres reprises, lors des élections générales d'avril puis complémentaires de juin à l'Assemblée constituante; surtout, ce scrutin met en lumière les interférences entre pouvoir local et pouvoir national, conjoncture nationale et contextes locaux, et la manière avec laquelle les catégories du débat politique national s'insinuèrent parfois dans la formulation et la construction des enjeux communaux. A Plougastel-Daoulas, Edouard Nicole écrit le 23 août une longue lettre au sous-préfet de Brest ; il y dénonce, au préalable, "les intrigues et les manoeuvres ouvertes du parti prêtre et légitimiste », avant de préciser: "L'expérience faite dimanche dernier prouve que nous avions raison de pétitionner. En effet, le parti réactionnaire légitimiste et prêtre l'a emporté grâce à ses dégoûtants procédés de corruption et de captation; il a réussi à faire nommer à son gré les trois chefs de l'administration municipale de notre commune $»^{14}$. La surveillance politique au village pouvait se faire quelquefois à travers une grille de lecture idéologique nationale à laquelle les élections municipales apportaient aussi leur caution.

Un chiffre : le conseil de préfecture statua sur la validité de 39 élections municipales en 1846, de 54 élections municipales en 1848 (20\%), de 16 seulement en 1852 . On peut imputer l'inflation des protestations qui accompagnent, en 1848, les procès-verbaux, à plusieurs raisons: un nouveau régime pétri de vertus qui garantissait davantage les droits des citoyens et prêtait attention à tout ce qui pouvait corrompre un suffrage devenu universel; une conjoncture politique plus ouverte qui permettait, pour un certain nombre d'impétrants au pouvoir municipal, de vider leurs querelles en faisant appel, en dernier recours, à l'autorité supérieure ; une tolérance moindre envers tout ce qui relevait de la fraude; une surveillance accrue des opérations électorales, une surveillance interne à la commune-communauté, dans un contexte mouvant et fluctuant où une nouvelle loi du nombre pouvait imposer tant de changements et de soubresauts. C'est cette dernière hypothèse que nous allons privilégier. Car les élections municipales sont un moment particulier, une étape décisive dans la construction d'une identité communale : moment d'unité, moment où la communauté peut éprouver son appartenance collective à une entité indivisible, l'élection municipale a été dès la monarchie de Juillet un catalyseur d'une unité territoriale encore jeune - les communes ne datent que de la Révolution - concurrencée par l'ancien maillage paroissial ${ }^{15}$. Confrontée aux forces centrifuges des hameaux - nous sommes dans un pays de bocage -, mise à mal par les revendications d'indépendance de ces mêmes hameaux, la commune a été souvent considérée comme un territoire prescrit plutôt que comme un territoire vécu ${ }^{16}$. Aussi, l'élection symbolisait-elle l'appartenance à un territoire administratif et politique qui excédait, pour l'individu, l'horizon plus quotidien d'un territoire borné par les activités économiques et les ancrages sociaux. Avec des changements en 1848 : l'universalité du suffrage permet à tous les hameaux - et ils peuvent être nombreux, plus d'une quarantaine parfois d'avoir des électeurs présents le jour du scrutin et donc, d'être potentiellement représentés au sein du conseil municipal, alors que le suffrage censitaire condamnait certaines portions du territoire communal au silence; cette extension du cadre politique local portait intrinsèquement en elle une transformation de l'équilibre territorial, un bouleversement plausible des positions de pouvoir que certains notables capacitaires avaient acquises au cours des deux décennies de la monarchie de Juillet... ou, tout au moins, un imaginaire de ce possible renouvellement.

En 1848, les élections municipales sont donc entourées d'une peur ou d'un espoir de changement, d'une illusion de la régénération et d'une rumeur de la désorganisation 
qui ont été autant de vecteurs de l'extrême surveillance endogène et pointilleuse des opérations électorales.

(Auto)surveillance politique au village et apprentissage de l'ordre démocratique

La surveillance politique au village en 1848, entendue ici comme une affaire interne à la commune-communauté et liée, en priorité, au moment électoral - une autosurveillance politique en quelque sorte -, est ainsi au coeur des scrutins communaux. Elle procède bien évidemment des réflexes de contrôle qui ont présidé à la mise en place des premiers scrutins communaux. Elle procède plus largement d'une habitude de surveillance liée à un univers villageois de l'interconnaissance, où la connaissance et la reconnaissance de l'autre, au-delà des simples proximités et des structures du voisinage, passent par le guet d'autrui ${ }^{17}$. Ainsi, dans cette société sous surveillance mutuelle, le regard porté sur l'autre permettait plus globalement de fixer et de figer des normes pourvoyeuses d'inclusion et d'exclusion ${ }^{18}$. Certains pourront arguer cependant que la surveillance politique se confondait tellement avec une attitude générale de contrôle réciproque systématisé qu'il est impossible d'en déterminer des contours bien spécifiques, de l'individualiser; et ceci serait d'autant plus vrai que ces ruraux ne faisaient guère de différence entre les exercices du contrôle social auxquels ils étaient soumis et qu'ils exerçaient eux-mêmes. A cela, on peut être tenté d'objecter la réponse qui suit : ce n'est pas parce qu'une société ne semble pas donner à voir une distinction, ce n'est pas parce qu'une société n'a pas vécu et ne s'est pas pensée à l'intérieur de la grille de lecture que lui impose le chercheur, que l'interprétation qu'il peut en faire est frappée d'anachronisme, bien au contraire ; poser des questions que des hommes, à une époque donnée, ne se posaient pas, est souvent un biais concluant pour tenter de comprendre les ressorts du fonctionnement social et, ici, de la fabrique de la procédure électorale. Ainsi, la surveillance politique se distingue, à notre avis, de la surveillance générale pour plusieurs raisons: elle a une morphologie qui lui est propre ; elle est inhérente à un mode de décision politique imposé par l'Etat - l'élection - et fonctionnant selon un cadre normatif particulier ; elle est ponctuelle puisqu'elle est liée à un moment électoral récurrent certes, mais exceptionnel cependant - les électeurs censitaires qui ont voté depuis 1831 n'en sont qu'à leur septième expérience municipale en 1848 -; enfin, elle connaît en 1848 un sommet, dans un moment où la fièvre électorale est porteuse de possibles changements.

Approfondissons. La surveillance politique a ses lieux: le bourg qu'elle permet de consacrer, elle aussi, épisodiquement, comme le coeur du pouvoir communal et le centre du territoire prescrit ${ }^{19}$. Elle demeure une surveillance en pointillé, avec ses propres rythmes qui coïncident avec la mise sous tension, lors des élections, de la communauté. Elle est, en cela, discontinue, segmentée. La surveillance politique peut ainsi débuter quelques jours avant le scrutin, lorsque des agents électoraux parcourent la campagne, menacent, font des promesses et distribuent des bulletins. Elle connaît son acmé au moment du vote, avec là encore des scansions qui attestent les heures cruciales du scrutin : lors de l'ouverture des élections, alors que la plupart des votants se pressent pour voter - on utilise encore, comme pour les élections générales, l'appel nominal, ce qui oblige fréquemment les électeurs à se présenter dès le début des opérations; au moment de la fermeture du scrutin qui prélude au dépouillement, alors que la salle qui a été le théâtre de la journée électorale se remplit de nouveau. Enfin, cette surveillance politique est profondément liée aux votants - cela va de soi -, et pour l'analyste, à un certain nombre d'individus qui apparaissent dans les protestations: ceux dont les auteurs des contestations, dans une sorte de mise en abyme de la 
surveillance, condamnent les tentatives d'influence et de prévarication électorale; ceux qui, dans une narration du moment électoral, décrivent moins par le menu les exactions perpétrées que les entorses aux règles imposées par la loi et la lecture qu'ils en font. Si l'autosurveillance politique est communément partagée par les électeurs, il est bien évident qu'elle connaît des seuils : on était d'autant plus sourcilleux sur le déroulement du scrutin que l'on avait des positions de pouvoir à défendre, à conquérir, et que l'on savait porter, par écrit, ses récriminations devant le conseil de préfecture. Ajoutons à cela un dernier élément déterminant: cette surveillance politique avait d'autant plus d'intérêt et de prise qu'elle enrichissait une connaissance fondée sur une surveillance plus quotidienne liée au voisinage ou aux proximités sociales; il va cependant sans dire que dans les grandes communes, la connaissance de l'autre lointain - celui qui était parfois à l'autre bout de la commune - relevait davantage d'un savoir empirique des alliances familiales, des généalogies et des parentèles, que d'une surveillance épisodique liée à un moment exceptionnel.

Dans cette société du contrôle social permanent, la surveillance politique semble obéir à deux logiques paradoxalement antagonistes : elle tend à légitimer le rôle des notables traditionnels - curés et maires, nobles, élites sociales de la commune - qui, inquiets devant la puissance du nombre et la possible remise en cause des hiérarchies traditionnelles, exercent un contrôle plus tatillon et plus visible qu'auparavant (ce faisant, ils créditent les élections, par leur présence et leur surveillance des urnes, d'enjeux politiques par ailleurs passés sous silence et niés par l'administration). Elle fait de chaque votant un citoyen qui se construit en tant que tel, puisque sa participation à la vie politique de la commune passe non seulement par son acceptation de la procédure électorale comme moyen de décision mais aussi par l'attention portée à la réciprocité des pratiques chez ses semblables.

Sur le second point - la fabrication de l'identité du citoyen-électeur au moment de son passage à l'acte de voter -, de nombreux travaux ${ }^{20}$ ont montré comment elle était profondément liée à la progressive acceptation d'une norme électorale en cours d'élaboration. Surveiller son prochain au moment où il vote et, a fortiori, contester la manière avec laquelle il a utilisé son bulletin ou la façon dont se sont déroulées les opérations, supposent que le scrutin obéisse à des exigences qui ne recouvrent pas forcément les valeurs et les combinaisons du pouvoir local ; qu'il obéisse à des règles édictées par l'Etat auxquelles doivent se conformer tous les hommes, quel que soit leur rang dans les hiérarchies sociales. L'autosurveillance politique structure ainsi le regard porté sur la scène électorale. Dans le Finistère, comme ailleurs, les élections de l'été 1848 n'échappent pas au mouvement progressif de sanctuarisation du lieu de vote, de clôture du lieu de vote, de forclusion de la violence liée à l'élection ${ }^{21}$; et peu importe que l'on dépose son bulletin dans une urne de fortune placée dans une salle de la mairie ou dans un appartement privé réquisitionné pour l'occasion, ce qui compte, c'est que des éléments considérés comme fondamentaux dans la mise en place d'un ordre électoral soient au-dessus de tout soupçon. D'où l'attention accordée dans les protestations à trois composantes de cette civilité électorale : la constitution du bureau et sa discipline de travail au cours de la journée ; l'urne de toutes les tentations; le dépouillement, instant crucial où tout semble pouvoir encore, pour certains tout au moins, se jouer. Les détails abondent qui disent la gamme des possibles en matière de corruption et surtout les normes qui président à leur intégration dans la lecture des infractions à la déontologie de l'élection. Guengat (257 électeurs), près de Quimper, reste en ébullition après la fermeture du scrutin ; le procès-verbal des élections signale 
167 bulletins et 149 paraphes sur la liste des votants. Une différence troublante que ne manque pas de faire remarquer, entre autres choses, un certain Bidon: "Monsieur le Maire lui-même, de qui, il n'a pas dépendu que les choses se passassent plus régulièrement, viendrait sûrement appuyer les griefs qui vont être exposés. L'on taira les brigues et manèges inqualifiables de quelques électeurs à l'extérieur de la salle des votes. Comme presque partout, malheureusement, les pratiques intimidatrices ont eu lieu jusqu'au seuil de l'appartement. L'on s'arrêtera à ce qui y paraît illégal: $1^{\circ}$ Les meneurs, qui, en cas d'insuccès, se réservaient de revenir contre les élections, ont fait qu'il n'y a eu aucun arrêté, aucune délibération rectificative de la liste, aucune délibération non plus, ni le vingt-huit, ni les jours précédents pour arrêter en définitive la liste des électeurs communaux ; (...) $3^{\circ}$ Vient le jour et la tenue des élections. Vraie cohue, absence de tout ordre. Ainsi le bureau n'a pas été constitué avant les opérations. Il ne s'y trouvait joint au Maire que deux individus, Pierre Le Goaër de Pellavon et Jean Le Quéau de Quillihouarn. Ce fait, que le procès-verbal en parle ou non, sera prouvé par tous, il est de notoriété publique. Il n'y a eu, pour parvenir au vote, aucun appel nominal. Cette mesure d'ordre conservatrice des droits de tous, a été remplacée par un désordre intolérable. Les meneurs, sans attendre cet appel indispensable, se sont approchés du bureau, et flanqués de leurs adhérents et des timides, ils ont déposé leurs billets en bloc dans le vase destiné à les recevoir $»^{22}$. Suit encore une moisson de reproches qui témoignent, en plus des frictions personnelles, d'un sentiment d'injustice et d'un sentiment d'exigence électorale dont des études récentes ont montré à quel point ils avaient pu varier et évoluer ${ }^{23}$. Concernant les opérations à Plouénan - 3280 habitants, 21 conseillers municipaux à élire, 721 électeurs et 599 votants, soit une participation de $83 \%$-, un électeur décrit par le menu la journée du 30 juillet: "Les élections municipales se sont distribuées à Plouénan en trois sections isolées et séparées l'une de l'autre de quatre hectomètres environ: chaque section avait ses scrutateurs et son bureau où se cueillaient les suffrages des électeurs. Devant procéder au dépouillement, les billets des deux sections, réunie l'une à la chapelle dite Kerellou, et l'autre réunie au local dit reliquaire, ont été, chose absurde! déménagés, portés dans un panier d'osier, sans être cachetés sous bandes, pour être rendus à la salle de la Mairie où devait se faire et où même s'est fait le dépouillement. A ne consulter que le maintien négligé de ces paniers, vous les eussiez pris pour un panier de fraises ou de pêches ${ }^{24}$. L'urne apparaît ainsi comme une frontière métaphorique entre le public et le privé et doit se dégager des emprises de l'arrangement qui gouverne des pans du monde social.

Les questions de la légalité du vote et de la légitimité des résultats sont ainsi au coeur d'une narration de la surveillance politique dont témoignent les protestations annexées aux procès-verbaux, dès la clôture du scrutin, ou les contestations reçues par le maire dans les cinq jours qui suivent. Cette écriture de la surveillance politique, toujours libellée en français, emprunte à un certain nombre de codes spécifiques: elle décompose le moment électoral, insiste sur le détail, le témoignage direct, l'appel à une cohorte de témoins qui fixent par écrit leurs récriminations; en général, les griefs sont numérotés, hiérarchisés puis commentés. Avoir $\mathrm{vu}^{25}$, avoir entendu dire, avoir été plusieurs à voir - ce que manifeste l'apposition des signatures - doit attester l'évidence des faits. A Crozon, le notaire Alavoine s'appuie sur ce qu'il a vu et sur ce qu'il a su, une façon de prouver que la surveillance politique n'est pas liée à l'unique regard individuel porté sur la situation mais à un écho polyphonique, seul capable d'embrasser l'ensemble de la scène électorale; il commence ainsi sa lettre : «Dimanche trente juillet, lorsque la troisième section était appelée à voter, j'ai vu l'un des membres du bureau retirer de la poche de son gilet trois billets (...)»; il poursuit: "J'ajouterai qu'il est également à ma connaissance que dans la deuxième section l'une des listes a été enlevée par un des membres du 
Bureau qui s'est absenté pendant plus d'une heure en la conservant avec lui $»^{26}$. Alavoine a vu puis il a su : ce glissement du voir au savoir, c'est celui qui permet à un individu de rassembler le maximum de griefs et de se positionner en tant qu'électeur incorruptible lassé de constater la corruption des élections. Dans une société où l'attestation de la preuve est un gage de la victoire sur l'autre - c'est ce qui fait le quotidien des juges de paix et des plaignants -, le souci d'exactitude est un critère de la bonne foi de l'auteur ; et l'investigation menée, au nom d'une certaine conception de la morale, un gage de la crédibilité des faits avancés. L'écriture de la surveillance politique incite aussi à tenter de comprendre ce qui heurtait ce regard politique et ce que celui-ci oubliait ou ne pouvait envisager: quels sont les faits qualifiants et ceux passés sous silence? De même, elle est parfois un scalpel qui permet de faire apparaître les équilibres sociaux masqués, de rattacher telle pratique corruptrice à tel habitus ${ }^{27}$. Elle incite à prendre en compte le substrat social et les conditions locales qui président à l'élaboration de ce regard politique : les normes de la plainte et, partant, de la surveillance exercée au moment du scrutin, diffèrent selon les individus et selon les communes, même si des dénominateurs communs existent - le repérage de l'individu étranger à l'assemblée électorale, en particulier. L'écriture de la surveillance politique se construit en fonction des normes prescrites et participe pleinement à la juridicisation du politique ${ }^{28}$. Combien d'annexes aux procès-verbaux, combien de protestations véhémentes qui font appel à tel ou tel article de la loi du 21 mars 1831 pour dénoncer ici, l'absence de la liste électorale dans le bureau de vote, là, les vacances du bureau, etc. Explicitement ou en filigrane, l'écriture de la surveillance politique est revendiquée, par les auteurs, comme une façon de se ranger dans le camp du droit, et en 1848, de la République : "L'on se bornera à ces seules mentions[5 griefs].Elles paraîtront assez graves pour annuler les élections communales de Guengat. Ce sera un bon exemple et un véritable acte de patriotisme " ${ }^{29}$ écrit Bidon à la toute fin de sa réclamation. Le bon citoyen est celui qui brise le silence au nom du droit et assène la preuve ; celui dont la surveillance n'a pas été prise en défaut et qui s'érige en gardien de la loi et de l'honneur bafoué de la communauté.

Mais il y a plus. L'écriture de la surveillance politique est un jeu d'écriture. Elle ne cesse de brouiller les pistes puisque c'est à chacun sa vérité. Une vérité polymorphe, hésitant entre une indignation sélective en face des forfaits commis et une débauche de rumeurs. Dans le labyrinthe des protestations, il est souvent difficile de démêler le vrai du faux. On écrit pour se conformer à la norme prescrite; on écrit aussi pour la travestir ou pour l'instrumentaliser à son profit. Un exemple: le procès-verbal de Plouégat-Moysan mentionne que les 331 électeurs de la commune (1 335 habitants) étaient appelés à se rendre à l'église pour exercer leur droit de vote ; 217 le firent $(65,5$ $\%)$. Une protestation signée par une trentaine d'électeurs et datée du 30 juillet incrimine les pratiques du maire sortant qui aurait, entre autres entorses à la règle nationale, fait « distribuer sous le sceau de son autorité, des bulletins écrits à l'avance sur un papier de couleur bleue, afin de pouvoir contrôler les citoyens qui devaient voter en faveur de la coterie recommandée par le Citoyen Maire $\aleph^{30}$. La riposte ne se fait pas attendre: une autre trentaine d'électeurs réfute la protestation tandis que le maire, lui aussi, répond à chaque grief : «art. 6 de la protestation. Les citoyens Mercier et Goasdoué ont dit au maire: ordonnez de déchirer les bulletins écrits, qu'ont en poche les électeurs, et distribuez-en de blancs. Il était évident que le maire ne pouvait obtempérer à cette demande, chaque électeur, était parfaitement maitre de son bulletin; du reste tous avaient eu le temps de faire lire par des tiers ce que contenaient leurs bulletins; si donc ils ne leur eussent pas convenus ils les eussent changés $\aleph^{31}$. Dans la course à l'opprobre, dans la transformation de la surveillance en de 
multiples faux bruits, seul un élément permet de tirer cette affaire au clair, les résultats : 217 votèrent; le premier élu obtenait 214 suffrages et le dernier 201. Or, la protestation est signée par plus de 30 électeurs qui disent tous avoir glissé un bulletin dans l'urne. Certains d'entre eux votèrent donc pour la liste bleue - la liste du maire avant d'apposer leur signature au bas d'une protestation qui arguait de la nullité de l'élection, au regard des nombreuses infractions commises pendant les opérations ${ }^{32} \ldots$ Enfin, l'écriture de la surveillance politique permettait de prolonger, au-delà de la proclamation des résultats, le trouble, la brouille et la rivalité entre clans rivaux. Accepter d'écrire que l'on avait assisté, ensemble, à des faits répréhensibles, c'était accepter de s'intégrer à un groupe capable, après le jour des élections, de semer le doute et de rester soudé. En prévision de l'avenir.

Sur le premier point - l'autosurveillance politique légitimait le rôle des notables traditionnels qui imposaient d'autant plus leur présence avant le scrutin et au moment du vote qu'ils pensaient, pour certains, avoir beaucoup à perdre-, deux constats s'imposent. Une première lecture (non critique) des archives incite fréquemment à considérer ces notables sous l'angle des faiseurs d'élection, sans foi ni loi. Après avoir fait distribuer des bulletins dans les jours qui précèdent l'élection, après avoir usé de leur influence sociale et de leur pression plus ou moins amicale - en jouant inopinément parfois sur la promotion d'une toute nouvelle communauté des égaux sur leurs fermiers, leurs domestiques ou sur leurs ouailles, ils exerceraient au moment $\mathrm{du}$ vote un contrôle politique plus prégnant que jamais ${ }^{33}$. Ce que semble confirmer l'impunité avec laquelle certains n'hésitaient pas à corrompre les élections et à tricher avec outrance. L'intimidation et le climat de peur sont largement dénoncés : à Mespaul (1 351 habitants, 12 conseillers municipaux à élire, 254 votants et 301 électeurs), protestations et contre-protestations alimentent la chronique post-électorale. Une demande d'enquête est faite dès le 4 août : "Attendu que les élections de la dite commune qui ont eu lieu le 30 juillet dernier, n'ont été que le résultat de la corruption, et de l'intimidation, qu'ainsi Louis Auffret du petit Kergauan, l'un des conseillers élus agissant tant pour assurer son élection que celle des onze autres membres qui ont obtenu une faible majorité, a acheté d'une grande quantité d'électeurs, leurs suffrages, qu'il a payés à prix d'argent, tant la veille des élections que le jour même de ces élections, que ce fait est de notoriété et de clameur publique, et pourra être prouvé par un grand nombre de témoins (...), que le même dit Auffret a cherché à intimider d'autres électeurs, à l'effet d'obtenir leurs suffrages, faits également faciles à prouver $\aleph^{34}$. S'insurgeant contre cette protestation, les conseillers nouvellement élus s'empressent de répliquer et signalent "que tous ceux qui ont signé le procès-verbal des réclamations dressé par le citoyen Salaün de Kertanguy sont presque tous des fermiers à ce propriétaire ou des gens à son service qui bien entendu n'auraient pas voulu attirer les disgrâces de leur maître et propriétaire en lui refusant des signatures qu'il leur a réclamées (...) » ${ }^{35}$. Les exemples abondent qui concernent des urnes bourrées, des tumultes organisés, des règles du jeu transformées - la fermeture du bureau de vote avant les trois heures d'ouverture prescrites par la loi, par exemple. Ces écarts à la norme électorale imposée ne cessent d'étonner tant ils sont grossièrement perpétrés. Ils semblent confirmer surtout que l'autosurveillance politique profitait à des notables qui ne s'embarrassaient guère des principes du code électoral et allaient aisément jusqu'à le subvertir puisqu'ils étaient, a priori, des maîtres du jeu incontestés; des notables dont le seul but était la confirmation de leur omnipotence et leur reconduction à la tête du pouvoir. En un sens, la corruption électorale n'existait pas puisque l'élection ne servait qu'à valider des positions de domination quasiment intangibles. 
Une seconde lecture permet cependant d'aller au-delà du simple constat d'une soumission répétée des dominés aux dominants, dont l'acte électoral réactualiserait en quelque sorte le lien de sujétion. Il est évident que ce qui se joue au moment des élections municipales, en plus du processus de dévolution du pouvoir communal, c'est un rapport de force, l'affirmation d'une possible reconfiguration des liens sociaux. Le bulletin de vote porte en lui une part invisible des relations sociales qui irriguent la communauté ; et c'est cette part invisible que l'autosurveillance politique permet de rendre effective. La présence autour de l'urne, les regards croisés sur le bulletin -regard $\mathrm{du}$ fermier sur son propriétaire qui surveille son billet et sa manière de bien voter rendent compte des enjeux multilatéraux qui se trament au moment des élections et du possible rééquilibrage des positions sociales. Le bulletin de vote ne sanctionne pas mécaniquement, obligatoirement, des rapports de force et des hiérarchies installées; s'il réaffirme bien souvent le primat du notable, encore faut-il que ce primat soit assorti de nouvelles conditions de recevabilité. Ainsi, si certains furent spoliés de leur choix de décision, d'autres, en revanche, surent jouer de ce droit de choisir - voter pour ou éliminer - dans l'optique d'une renégociation des rapports sociaux ${ }^{36}$. A CloharsFouesnant, le scrutin municipal de l'été a alimenté une chronique électorale extrêmement volubile. L'affaire tourne autour des pressions et des moyens d'intimidation qui auraient servi au maire sortant, Hernio, pour s'assurer une victoire haut la main. Deux partis s'affrontent. On est pour ou contre Hernio, et à travers lui, pour certains tout au moins, pour ou contre la République. Parmi tous les griefs, l'un semble avoir mis le feu aux poudres. Le clan des protestataires s'en explique : «Outre les moyens ordinaires d'intimidation employés pour arracher des billets et pour obliger à voter comme lui, monsieur le président du Bureau ouvrait les billets à mesure qu'on les lui remettait, voyaient ceux qui étaient pour lui et inscrivaient le nom des personnes qui ne votaient pas sa liste $»^{37}$. L'enquête permet de faire la lumière sur les faits avancés. Le gendarme qui surveillait les élections, à la demande du maire qui craignait des incidents, confirme la version des protestataires; on peut ainsi lire dans la déposition : « il assure que les billets blancs étaient examinés par Mr Hernio, avec plus d'attention que les autres billets; il pense que Mr Hernio pouvait lire les billets de la manière dont il les ouvrait, qu'il prenait le nom des électeurs aux billets blancs et que plus tard il dit à son fils : tiens, voilà les noms de ceux qui nous ont manqué $\aleph^{38}$. Les 15 électeurs qui avaient mal voté selon Hernio, et que l'on peut imaginer en proie à la vengeance du maire, n'étaient-ils que des électeurs candides ? C'est ce que l'on peut supposer. Mais on peut aussi suggérer que conscients du prix de leur bulletin, ils s'étaient "vendus" peut-être à un autre homme d'influence plus offrant et plus généreux. Ou encore, que leur acte d'opposition relevait moins d'un vote ballotté entre des partis antagonistes que d'une volonté clairement affichée de conditionner leur adhésion à l'instauration d'un nouvel équilibre social. Ce qu'accrédite la déposition du quinzième témoin de l'enquête du 3 septembre 1848: "Gouin Jean, dépose qu'il avait eu d'abord un billet de Mr Perrotin, mais que L'haridon garde de Mr Hernio lui vint offrir un autre, qu'il lui répondit: je ne puis le prendre parce qu'il ne serait pas de la même couleur. Alors L'haridon lui remit un billet blanc en lui disant que de cette manière on ne saurait pas pour qui il avait voté; et qu'il remit en définitive au président le billet qu'il avait reçu de Mr Perrotin. Billet qui n'a pas été ouvert en le recevant $»^{39}$. Ainsi, Jean Gouin n'apparaît pas ici comme l'homme lige qu'il aurait ou avait pu être. Lors du marché électoral, au cours des tractations et des transactions qui précédaient le scrutin, puis au moment fatidique du vote, les résultats n'étaient pas forcément acquis par avance. Défier une autorité restait possible; et glisser son bulletin dans l'urne était alors là pour le prouver. 
La possible réactualisation des liens sociaux, leur possible recomposition sont donc le produit de l'autosurveillance. Ce n'est pas le moindre des paradoxes: le vote secret devait être visible et vu pour que l'individu existe et soit replacé dans la chaîne sociale. Déposé au vu et au su de tous, objet de manipulations possibles ${ }^{40}$, preuve immédiate de l'approbation ou de la désapprobation, le bulletin de vote témoignait, aux yeux de la communauté des votants, d'une allégeance acceptée ou d'une opposition désormais proclamée. Aussi, l'impossible secret du vote ne rimait pas forcément avec une confiscation du pouvoir par une sanior pars; le soutien apporté à l' "élite " politique locale était mesuré et mesurable puisqu'il était montré et attesté. De la même façon, la surveillance des notables, leur entrée physique dans la lice, la descente de leur Aventin en disaient long sur leur peur sociale, leurs difficultés à appréhender une situation mouvante, parce que nouvelle, dont la maitrise risquait de leur échapper. Devenus des citoyens comme les autres ou presque, ils se devaient, aux yeux de tous, d'user de leur entregent pour conquérir les suffrages. Concurrencés sur le terrain des pressions et de l'influence, ils participaient, contre leur propre volonté peut-être, à la formation d'un marché politique ${ }^{41}$ traversé par des clivages et surtout, à une décompositionrecomposition de leur statut politico-symbolique. 1848 marque, sans conteste, une nouvelle étape dans le consentement à la domination. Le châtelain n'est pas récusé à condition qu'il s'expose lui aussi, l'élection au suffrage universel permettant de mettre en lumière les rapports de force et de classes. Être considéré comme le maitre supposait dorénavant que l'on accepte de se montrer sous ces habits-là. A Nizon, Cyprien Hersart de la Villemarqué, 35 ans, gros propriétaire dans la commune, souhaite devenir maire à l'issue du scrutin de l'été 1848 ; l'un de ses parents lui a déjà prodigué des conseils : «ne manquez pas l'occasion, saisissez le pouvoir qui s'offre à vous, et quand vous en serez investi, faites tout ce qu'il faut pour le conserver et le transmettre un jour à un fils que Dieu vous accordera (...). Mettez beaucoup du vôtre dans votre administration, vous n'avez que du bien à faire et il est si doux d'en faire à tout ce qui nous entoure. Ces paysans de Nizon sont doux et dociles. (...) Pour cela, mon bon ami, il faut de l'aménité même un peu avec les habitants de PontAven, qui auront toujours une certaine influence sur les gens de Nizon. Vous dont le coeur est si bon, vous devez vous faire aimer de ces deux communes. Je veux que tous ces gens quand vous passerez disent : voilà notre protecteur et notre bienfaiteur $»^{42}$. Témoignage de la conviction que l'appropriation du pouvoir exigeait désormais des compromis, qu'il fallait se faire accepter pour ne plus apparaître comme le châtelain arrogant et méprisant de l'Ancien Régime ${ }^{43}$, cette lettre prend une autre dimension dès que l'on se penche sur la situation électorale de Nizon à la fin juillet 1848. Car le scrutin ne fut pas, en effet, vierge de tout incident, loin s'en faut. La protestation de l'ancien maire de la commune détaille les errements électoraux de la journée du 30 juillet ; on peut y lire ainsi : «le citoyen Cyprien De Lavillemarqué, frère du premier, m'a tenu les propos les plus grossiers; il a été jusqu'à me reprocher d'avoir obligé un nommé Portal à acquitter en journées de prestations, plus qu'il devait légalement. (...) Le citoyen Théodore De Lavillemarqué s'étant trouvé obligé de quitter la salle, s'est posté à la porte de l'assemblée où tantôt assis, tantôt debout sur une chaise, il forçait en quelque sorte les électeurs qui entraient à recevoir des billets de lui ; on l'a vu mettre la main dans la poche d'un ou deux électeurs et déchiré publiquement les bulletins d'un grand nombre d'électeurs, à qui je les avais remis sur leur demande, leur disant qu'ils étaient mauvais ${ }^{44}$. L'enquête demandée par le protestataire et organisée le 7 septembre 1848, après accord du conseil de préfecture, permet de voir défiler 13 comparants et de mieux déterminer les enjeux et les initiatives liés à l'élection, de cerner l'activité fébrile et brouillonne de Cyprien Hersart de la Villemarqué: "Le Citoyen Cyprien De Lavillemarqué a avoué qu'il 
avait engagé les électeurs de sa connaissance à se rafraîchir, mais qu'il n'avait pas vu en cela de fait de corruption, la dépense s'étant élevée au plus à cinq centimes par personne, et se voyant autorisé par l'exemple du Maire qui agissait de même $»^{45}$. Il devenait donc nécessaire pour un certain nombre de membres des élites traditionnelles de venir défendre sur le terrain leur pouvoir économique, social et symbolique, pour espérer guigner une place au conseil municipal. Entretenant eux aussi l'autosurveillance politique au village, se mêlant activement aux fièvres électorales, ils accréditaient davantage l'idée selon laquelle être élu supposait de "mener campagne", de se faire voir et d'être vu pour être reconnu. La seule domination sur une commune à partir du château était une période révolue. Il fallait donc participer à la réciprocité des regards, à cette journée où la commune-communauté éprouvait sa force et ses divisions devant ses membres. Ou les autorités traditionnelles s'y pliaient, ou elles risquaient parfois d'être abruptement contestées voire déboulonnées.

L'Etat en creux : surveillance politique et contrôle de l'Etat au village

Le peu d'intérêt apparent accordé aux élections municipales par l'administration ne doit cependant pas faire oublier que la mise en place d'un ordre électoral et démocratique se double d'un contrôle étatique en direction des communautés villageoises, puisque le vote est intrinsèquement une technologie de l'Etat. Un contrôle en mineur, manquant au prime abord de visibilité, car la surveillance politique qui s'exerce au moment des élections semble s'inscrire dans le seul cadre d'un contrôle social interne à la communauté. En n'obligeant pas les instances municipales à encadrer le suffrage, grâce à l'utilisation des institutions du maintien de l'ordre, l'Etat paraissait confirmer que l'élection locale relevait des joutes de clocher à circonvenir dans le seul cas où elles risquaient de mettre en danger durablement l'existence de la communauté et son unité. Ce désengagement - ou plutôt ce non-engagement - de l'administration contraste singulièrement avec le processus d'une emprise accrue du pouvoir central sur la société française que les différents régimes, au cours du XIXème siècle, n'ont cessé de promouvoir. On serait alors tenté de considérer ce nonengagement comme la preuve d'un désintérêt manifeste pour des conflits dont la teneur risquait d'échapper à n'importe quel observateur extérieur, aussi sagace fût-il.

Ce serait peut-être faire fausse route. L'administration n'était pas interventionniste car elle savait que son intrusion dans les affaires communales risquait de compliquer les choses, voire d'alimenter un sentiment de défiance à l'égard des autorités extérieures ${ }^{46}$. Elle était d'autant moins interventionniste qu'elle n'avait pas besoin de l'être: les remous et les rumeurs électorales lui parvenaient très rapidement et il ne lui était pas nécessaire de s'immiscer dans les affaires communales. Surtout, l'élection, comme mode de dévolution du pouvoir à l'intérieur de la commune, n'a été ni rejetée ni concurrencée par une autre norme d'accès aux responsabilités municipales, bien au contraire. A la différence des autres formes d'encadrement (tutelle financière, tutelle de la gendarmerie), la mise en place d'un ordre démocratique au village n'a pas été contestée ; et l'on ne saurait trop lier cette acceptation de l'élection au seul fait que la société finistérienne apparaisse en priorité comme une société d'obéissance et non comme une société de dissidence ${ }^{47}$. L'élection a intéressé et des individus et les communautés. Enfin, l'acceptation de la procédure électorale avait pour corollaire l'inoculation progressive d'une norme de référence avec laquelle les mécontents pouvaient jouer mais qui n'était pas remise en cause pour elle-même; dans les protestations, les renvois aux articles de la loi du 21 mars 1831 sont innombrables et c'est derrière elle que s'abritent de nombreux contestataires pour essayer de donner du 
poids à leur témoignage et à leur argumentaire, dans l'espoir de faire basculer la balance du "tribunal administratif » en leur faveur. Car l'ultime recours, quand une affaire est portée sur la place publique, reste bel et bien le conseil de préfecture ${ }^{48}$. Sa légitimité, il la tire à la fois de son lien organique avec les services de la préfecture, de sa capacité de dire le droit, de son aptitude à faire la lumière sur une affaire - et donc de trancher--, de sa faculté de contester un ordre politique communal qui ne serait que le décalque d'un ordre social... et des affaires qu'il eut à juger. Son incorruptibilité ne résiste pas à l'épreuve de certains témoignages et il serait des plus utiles de subordonner l'étude des décisions qu'il prit et la fabrique des normes électorales qu'il promut à l'examen attentif des membres qui le composèrent ${ }^{49}$. Les liens politiques, les réseaux familiaux, l'appartenance à des mêmes univers sociaux, eurent en effet raison d'une pratique de la décision arbitrale fondée uniquement sur les seuls critères du droit et de la jurisprudence. Ainsi, dans l'affaire de Nizon, Théodore Hervé Marie de Kernaflen de Kergos (1779-1858), un parent des Hersart de la Villemarqué, écrivait au futur maire de Nizon le 26 août 1848 : «je crains bien que vos élections ne soient cassées. Théodore a vu deux fois le préfet qui agit peu. Il n'a cependant pas de mauvais vouloir, mais il attend lui-même à chaque moment sa destitution. Le Ster qui était pour nous dans le conseil de Préfecture vient d'être destitué. Les autres membres sont contre nous et veulent une enquête. Le préfet déclare qu'il ne la veut pas et dit qu'il tachera d'arranger cela. Mais le pourra-t-il ? Il traîne en longueur. Cela ne vous est pas désavantageux $\aleph^{50}$. Que retenir de cette lettre? L'existence de pressions sur un conseil de préfecture et la collusion possible entre des membres appartenant aux mêmes milieux sociaux; un arbitrage de ce tribunal administratif avant la lettre, plus enclin à se prononcer en fonction des enjeux politiques et des querelles de personnes qu'à se déterminer au nom d'une logique juridique patiemment élaborée; enfin, et surtout, l'attente d'une décision extérieure qui condamnait le citoyen Hersart de la Villemarqué à être un citoyen comme un autre, dont le succès de l'entreprise électorale était conditionné non plus à son emprise symbolique et sociale sur sa commune, mais à un arrêté de validation de son élection. Il y eut enquête, comme nous l'avons vu. Les élections furent cassées par le conseil de préfecture, lors de sa séance du 15 septembre, au nom de la violation manifeste de "l'esprit de loyauté \& d'indépendance qui doit présider à l'exercice d'un droit aussi sacré que le droit électoral $\aleph^{51} \ldots$ et peut-être, plus souterrainement, en fonction d'inimitiés personnelles qui, par ailleurs, nous échappent. On vota donc de nouveau le 8 octobre de 7 heures 30 à 11 heures. La liste de Cyprien Hersart de la Villemarqué passa en totalité52 ${ }^{52}$ Personne ne fit appel. La netteté de la victoire fit-elle oublier les incartades passées? Rien n'est moins sûr. Et l'on peut imaginer sans peine que pour quelques-uns, la revanche se préparait à l'ombre de l'éventuelle saisine de l'assemblée quimpéroise. Ainsi, le conseil de préfecture comme recours et expert permettait d'installer une surveillance, certes molle, mais possiblement réceptive aux arguments dénonçant le mauvais déroulement des élections. Abstraite, lointaine, cette justice administrative n'en incarnait pas moins ce niveau supra-communal de la surveillance politique qui, au lieu de s'opposer à l'autosurveillance villageoise, s'adossait à elle pour mieux en amplifier les effets. Recourir au jugement du conseil de préfecture, c'était accepter, involontairement peut-être, la tutelle souple de l'Etat ${ }^{53}$.

Cette surveillance administrative en mineur, cette surveillance de référence, acceptable par les communes justement parce qu'elle ne semblait ni odieuse ni contraignante ni en opposition avec l'autosurveillance entretenue au cours des élections, a permis en outre de promouvoir davantage la figure du maire. Président du bureau de vote, caution du 
bon déroulement du scrutin, c'est vers lui que les regards se tournent quand il s'agit de donner la version administrative des élections. Rarement, il est désavoué par le conseil de préfecture, à moins qu'un règlement de compte ne vienne soudainement influer sur le cours de la décision. Aux protestations d'un ou de plusieurs votants fait presque toujours écho une lettre du magistrat, écrite à la demande du sous-préfet ou, plus généralement, de sa propre initiative. En plus du procès-verbal des élections, elle est le document privilégié par le conseil de préfecture pour approuver ou condamner les élections. Le pacte de confiance instituant une relation privilégiée entre le maire et l'administration supérieure, et qui faisait du magistrat municipal un maillon essentiel du système administratif, transparaît dans de nombreux avis du conseil de préfecture. Ce dernier entérine généralement les explications du maire concernant le déroulement des élections, comme en témoigne, par exemple, l'affaire de Plonéis. Le 2 août, une protestation série les griefs, pointe l'absence d'affichage de la liste électorale dans la salle du scrutin, l'écart entre le nombre de suffrages contenus dans l'urne (112) et le nombre d'émargements (95). Le 5 août, le maire fait parvenir à la préfecture le procèsverbal des élections, joint la protestation et rétorque point par point : «Pour le second grief relatif au désaccord remarqué entre le nombre des votants et des émargements, on peut vérifier qu'il se réduit à 5 ou 6 , ce qui n'est pas étonnant à la campagne où il existe peu de personnes capables de remplir les fonctions de scrutateur avec une exactitude infaillible $"^{54}$. Le 18 août, le conseil de préfecture statue, « $V u(. .$.$) la protestation présentée par onze citoyens$ de la commune de Plonéis » et " $V u(. .$.$) les explications fournies par le Cen Maire de Plonéis »;$ reprenant l'argumentaire du maire ${ }^{55}$ et le créditant ainsi d'une vérité des faits, il valide les résultats. Ainsi, son rôle de surveillant en chef fait accéder progressivement le premier magistrat de la commune à un statut de plus en plus ambivalent : porte-parole de sa commune-communauté, garant de l'honneur de cette dernière, il se situe inexorablement dans une position d'intermédiaire entre l'Etat et elle. La surveillance politique tend à l'extraire de la gangue communautaire pour lui faire endosser les habits de l'agent de l'Etat et en faire un porte-parole du droit; une façon pour l'administration supérieure de promouvoir un ordre administratif supérieur aux intérêts de la communauté en s'appuyant sur celui qui incarnait, de plus en plus, la surveillance politique légitime au village.

La surveillance politique ne s'exprime pas, au village, au moment des scrutins locaux, selon le schéma classiquement mis en avant d'un encadrement systématique et contraignant des opérations par des forces étatiques du maintien de l'ordre. C'est ce que nous avons essayé de montrer. En revanche, si l'élection crée de fait les conditions d'une autosurveillance politique, la surveillance au village, quotidienne, le contrôle social fait de réciprocité se nourrissent aussi de cette autosurveillance politique qui permet à la commune-communauté de se revendiquer un peu plus comme une unité territoriale et humaine singulière. Ainsi, l'autosurveillance politique au village, outre qu'elle légitime le principe de l'élection et ses modalités, conforte ou infirme des hiérarchies sociales, et contribue, dans un moment d'incandescence électorale, à l'affirmation d'une identité communale.

Cette surveillance politique endogène s'insère cependant dans le cadre d'une norme administrative extérieure qui sert de référence en cas d'incident voire de conflit. Le citoyen protestataire devient un citoyen qui accepte implicitement ou explicitement les règles de l'élection, quand bien même il utilise les dites règles à ses propres fins. Progressivement et avec des rythmes différents, le référent législatif national - dont le conseil de préfecture était le premier garant -, discuté mais rarement contesté, s'inscrit 
ainsi dans l'horizon politique au village et marque une emprise accrue de l'Etat sur le monde social. L'autosurveillance politique participe sans conteste d'un double phénomène de construction: la construction d'un ordre démocratique et celle d'un ordre étatique, par le biais d'une imposition tacitement acceptée de la norme - même si celle-ci s'est trouvée amendée en fonction des situations.

L'autosurveillance politique est ainsi au coeur des scrutins communaux. Dans ce jeu des regards, dans les dénonciations des tricheurs et les contestations des élections truquées, c'est d'une intériorisation des normes et d'une domestication des pratiques électorales qu'il s'agit. En ce sens, les élections de l'été 1848 marquent une étape non négligeable dans la distinction qui s'opère entre l'homme privé, inscrit dans son réseau d'interrelations familiales et sociales, et le citoyen-électeur, spectateur, acteur et parfois contempteur d'un ensemble de règles qui ressortissaient à une pratique électorale imposée par l'Etat, et dont la surveillance lui incombait au niveau local. Dans cet univers de l'autosurveillance, la surveillance individuelle de la geste électorale a contribué, indubitablement, au renforcement de la chose politique.

\section{NOTES}

1. . La lecture des travaux de Michel Foucault a été d'un inépuisable secours, en particulier : Surveiller et Punir. Naissance de la prison, Paris, Éditions Gallimard (coll. Tel), 1993 (1ère édition, 1975), pp. 174-175, 202, notamment ; Dits et écrits, 1954-1988, Paris, Éditions Gallimard (coll. Quarto), 2001, volume $1: 1954-1975$, p. 1181 («On sait bien que ce ne sont pas les gouvernants qui détiennent le pouvoir. (...) 'Dominer', 'diriger', 'gouverner', 'groupe au pouvoir', 'appareil d'État', etc., il y a là tout un jeu de notions qui demandent à être analysées. De même, il faudrait bien savoir jusqu'où s'exerce le pouvoir, par quels relais et jusqu'à quelles instances souvent infimes, de hiérarchie, de contrôle, de surveillance, d'interdictions, de contraintes. Partout où il y a du pouvoir, le pouvoir s'exerce » notait-il dans un entretien avec Gilles Deleuze), pp. 1334-1336, 1473 ; volume 2 : 1976-1988, pp. 74-76, 629, 836-838.

2. . Bourdieu P., Propos sur le champ politique, Lyon, Presses Universitaires de Lyon, 2000, pp. 51-69 en particulier.

3. . Thématiques au coeur des analyses d'Alain Garrigou, Histoire sociale du suffrage universel en France, 1848-2000, Paris, Éditions du Seuil (coll. Points Histoire), 2002.

4. . Recueil des actes administratifs de la préfecture du département du Finistère, Quimper, Imprimerie de Lion, 1848, p. 273.

5. . Lagoueyte P., « Apprentissage et incidents électoraux à l'aube du suffrage universel : le scrutin d'avril », dans Bourdin P., Caron J.-C. et Bernard M. (dir.), L'incident électoral de la Révolution à la Vème République, Clermont-Ferrand, Presses Universitaires Blaise-Pascal, 2002, pp. 101-119.

6. . Archives nationales, C 1404.

7. . C'est nous qui soulignons.

8. . Archives nationales, C 1404. 
9. Sur la gendarmerie, on pourra se reporter à la très complète note de lecture de Douki C., «Entre État et société : la gendarmerie au XIXème siècle », dans la Revue d'histoire moderne et contemporaine, tome 50, $\mathrm{n}^{\circ} 1$, janvier-mars 2003, pp. 189-197 ; Thibon C., «L'ordre public villageois : le cas du Pays de Sault (1848-1914) », dans Maintien de l'ordre et polices en France et en Europe au XIXème siècle. Actes du colloque de Paris et Nanterre, 8-10 décembre 1983, organisé par la Société d'histoire de la révolution de 1848 et des révolutions du XIXème siècle, Paris, Éditions Créaphis, 1987, pp. 309-325.

10. . Archives départementales du Finistère (A.D.F.), 3 M 175, protestation du 6 août 1848.

11. . A.D.F., 3 M 172, lettre du maire au préfet, 9 août 1848.

12. . Par extension, et parmi une très abondante bibliographie, des réflexions dans : Karnoouh C., « La démocratie impossible. Parenté et politique dans un village lorrain », dans Études rurales, n 52, octobre-décembre 1973, pp. 24-56 ; Hervieu B., "'Le pouvoir au village' : difficultés et perspectives d'une recherche ", dans Études rurales, n 63-64, juillet-décembre 1976, pp. 15-30 ; Le Guirriec P., Le pouvoir en campagne. Formes locales du politique en Bretagne, Rennes, Éditions Apogée, 1994 ; Atrux M., La politisation des campagnes lyonnaises au XIXème siècle : l'exemple de Collonges-au-Mont-d'Or, Mémoire de maîtrise (sous la direction de Jean-Luc Mayaud), Université Lumière-Lyon 2, 2002. 13. . Guionnet C., L'apprentissage de la politique moderne. Les élections municipales sous la monarchie de Juillet, Paris, Éditions L'Harmattan, 1997.

14. . A.D.F., 3 M 180, lettre du 23 août 1848.

15. . Voir les premiers chapitres (en particulier ceux consacrés par Gérard Sautel à la Révolution française et par Philippe Vigier à la monarchie de Juillet puis à la Seconde République) du livre collectif sous la direction de Fougère L., Machelon J.-P., Monnier F., Les communes et le pouvoir de 1789 à nos jours,Paris, Presses Universitaires de France, 2002 ; une approche locale dans Kermoal C., Les notables du Trégor. Éveil à la culture politique et évolution dans les paroisses rurales (1770-1850), Rennes, Presses Universitaires de Rennes, 2002, pp. 321-363 plus particulièrement.

16. . Sur la question de l'identité communale, des approches différentes dans : Morel A., «L'espace social d'un village picard », dans Études rurales, n 45, janvier-mars 1972, pp. 62-80 ; Maresca S., « Le territoire politique », dans la Revue Française de Science Politique, volume 34, n³, juin 1984, pp. 449-466 et pp. 456-457 particulièrement ; Vivier N., Propriété collective et identité communale. Les biens communaux en France 1750-1914, Paris, Publications de la Sorbonne, 1998 ; Lefebvre H., La production de l'espace (4e édition), Paris, Éditions Anthropos, 2000, pp. 48-50 ; Frémont A., La région espace vécu, Paris, Éditions Flammarion (coll. Champs), 1999, pp. 75-84 et pp. 176-179 ; Ploux F., Guerres paysannes en Quercy. Violences, conciliations et répression pénale dans les campagnes du Lot (1810-1860), Paris, Éditions de La Boutique de l'Histoire, 2002, pp. 127-160 et pp. 182-191. 17. . Ce qu'a parfaitement montré, par exemple, Annick Tillier dans : Des criminelles au village. Femmes infanticides en Bretagne (1825-1865), Rennes, Presses Universitaires de Rennes, 2001. Le contrôle des corps et des gestes des femmes enceintes ressortit ici, à la fois à l'honneur des femmes, des familles, et de la société villageoise dans son entier. Voir aussi, dans une perspective plus historiographique, les pages que consacre Gaston Roupnel dans son Histoire de la campagne française, Paris, Plon, 1974, (1ère édition : Bernard Grasset, 1932), pp. 327-333.

18. . C'est ce qui apparaît en filigrane dans les travaux de : Claverie E. et Lamaison P., L'impossible mariage. Violence et parenté en Gévaudan, 17ème, 18ème et 19ème siècles, Paris, 
Éditions Hachette, 1982 ; Pourcher Y., Les maitres de granit. Les notables de Lozère du XVIIIème siècle à nos jours, Paris, Éditions Olivier Orban, 1987.

19. . Des remarques suggestives dans l'article de Marc Abélès, « Anthropologie des espaces politiques français », dans la Revue Française de Science Politique, volume 38, n5, octobre 1988, pp. 807-817.

20. . Garrigou A., Histoire sociale du suffrage universel en France, 1848-2000, ouv. cité ; Gueniffey P., Le nombre et la raison. La Révolution française et les élections, Paris, Éditions de l'École des Hautes Études en Sciences Sociales, 1993, pp. 323-428 en particulier ; Ihl O., "Tours de main et double jeu. Les fraudes électorales depuis la Révolution française ", dans Poirmeur Y. et Mazet P. (dir.), Le métier politique en représentations, Paris, Éditions L'Harmattan, 1999, pp. 51-87 ; Joignant A., « Un sanctuaire électoral. Le bureau de vote et l'invention du citoyen-électeur au Chili à la fin du XIXème siècle ", dans Genèses, $\mathrm{n}^{\circ}$ 49, décembre 2002, pp. 29-47.

21. . Déloye Y., Ihl O., «La civilité électorale : vote et forclusion de la violence en France ", dans Philippe Braud [dir.], La violence politique dans les démocraties européennes occidentales, Paris, Éditions L'Harmattan, 1993, pp. 75-96.

22. . A.D.F., 3 M 174, protestation du 3 août 1848.

23. . On lira avec intérêt, outre les différentes communications du colloque de Clermont-Ferrand, la conclusion de Jean-Claude Caron dans : L'incident électoral de la Révolution à la Vème République, op. cit., pp. 299-307.

24. . A.D.F., 3 M 180, lettre du 6 août 1848.

25. . Le témoin oculaire est omniprésent dans les opérations électorales de Mespaul. L'élection est affaire de regards concurrents, comme on peut le lire dans les récriminations datées du 28 septembre $1848:$ : $3^{\circ}$ Attendu que des électeurs ont été gardés à vue après qu'on leur avait distribué des bulletins jusqu'au moment où ils les ont déposés dans l'urne (...) », A.D.F., 3 M 178. Ces regards croisés obéissent à une logique de la " garde à vue » propre à assurer la véracité des situations dénoncées par écrit.

26. . A.D.F., 3 M 172, lettre du 3 août 1848.

27. . A Lopérec, la figure du notaire véreux, rapace, mettant en coupe réglée la commune, s'inscrit dans une dialectique populaire de l'abus de pouvoir commis en toute impunité par les " gros » à l'encontre des " petits » et de la révolte qui pouvait, en dernier recours et contre une telle emprise, gronder. La déposition du maire, paysan et propriétaire, ne cesse de corroborer un stéréotype dont l'étude mériterait d'être faite. Jean Rolland stipule ainsi le 23 août 1848 «Que le citoyen Menn Pierre-Louis, Notaire, demeurant au chef-lieu de la commune de Lopérec voyant qu'il avait échoué dans l'espoir de rentrer dans le conseil municipal et voyant que le dépouillement ne pouvait se terminer le premier jour, le lendemain avant le jour, il monte à cheval, parvient à force de ruses, de dol et de menaces, de gagner une centaine de personnes en grande partie ne sachant ni lire ni écrire, et de les intimider par des paroles déplacées s'ils ne venaient au bourg le lendemain et ce sans s'expliquer d'avantage... Ces gens se sont rendus chez le dit Citoyen Le Menn balançant entre le doute et la crainte ne sachant pourquoi on les appelait; le dit Le Menn pour mieux jouer son rôle commence par leur distribuer de la boisson au point de les rendre ivres, ce forfait terminé contre le droit des gens, contraire au principe de liberté et offusquant grièvement le principe de fraternité, il finit par leur distribuer des billets en son nom qu'ils ont déposés dans l'urne ne sachant encore ce qu'ils faisaient et ignorant complètement ce qu'ils allaient devenir », A.D.F., 3 M 174.

28. . Willemez L., « Le droit dans l'élection. Avocats et contestations électorales dans la France de la fin du Second Empire », dans Genèses, n 46, mars 2002, pp. 101-121. 
29. A.D.F., 3 M 174, protestation du 3 août 1848.

30. A.D.F., 3 M 180, protestation du 30 juillet 1848 .

31. . A.D.F., 3 M 180, note non datée en réponse à la protestation du 30 juillet 1848.

32. . Une incohérence de calcul qui n'échappa guère au scrupuleux sous-préfet de Morlaix. Dans son avis concernant les élections de Plouégat-Moysan, il notifiait le 21 août 1848 : «Considérant que les motifs énoncés dans la dite protestation ont été réfutés d'une manière suffisante et que d'ailleurs le grand nombre de voix qu'ont obtenues les élus (l'unanimité à bien dire) font penser que la majeure partie des signataires de la protestation ont pu voter pour eux », le conseil de préfecture se devait de valider les élections, A.D.F., $3 \mathrm{M}$ 180.

33. . Garrigou A., "Clientélisme et vote sous la IIIème République », dans Briquet J.-L. et Sawicki F. (dir.), Le clientélisme politique dans les sociétés contemporaines, Paris, Presses Universitaires de France, 1998, pp. 39-74.

34. A.D.F., 3 M 178, protestation du 4 août 1848.

35. . A.D.F., 3 M 178, protestation du 23 août 1848.

36. . Voir, pour une autre époque, les réflexions stimulantes de Giovanni Levi sur la plasticité et la morphologie du pouvoir dans une communauté rurale : Le pouvoir au village. Histoire d'un exorciste dans le Piémont au XVIIème siècle, Paris, Éditions Gallimard, 1989 (traduction française de l'ouvrage publié en Italie en 1985), pp. 140-142 notamment.

37. A.D.F., 3 M 172, protestation du 3 août 1848.

38. . A.D.F., 3 M 172, procès-verbal d'enquête du 3 septembre 1848.

39. . A.D.F., 3 M 172, procès-verbal d'enquête du 3 septembre 1848.

40. . A Plounévez-Lochrist, les protestataires écrivent le 2 août 1848 : « Chacune de ces sections ayant voté dans la même salle, et tous les bulletins ayant été déposés dans la même urne, il en est résulté une perte énorme de temps, et de plus une confusion qui a été bien funeste à la liberté de vote; en effet, pendant les opérations, les électeurs des trois sections confondus ensemble dans la salle encombraient tellement l'appartement, qu'un grand nombre d'entre eux, dans l'impossibilité d'arriver jusqu'au bureau, faisaient passer leur bulletin de main en main au président, livrant ainsi aux autres le secret de leur vote », A.D.F., 3 M 181.

41. . Phélippeau E., L'invention de l'homme politique moderne. Mackau, l'Orne et la République, Paris, Éditions Belin, 2002.

42. . Archives privées De la Villemarqué de Cornouaille, lettre du 18 avril 1848.

43. . Brelot C.-I., La noblesse réinventée. Nobles de Franche-Comté de 1814 à 1870, 2 tomes, Besançon, Annales littéraires de l'Université de Besançon, 1992, tome 1 : Restaurations et reconversions, pp. 562-579; du même auteur, « Le Château face au vote paysan (1800-1882) », dans Politix, n 15, 3e trimestre 1991, pp. 53-58.

44. A.D.F., 3 M 178, protestation non datée.

45. . A.D.F., 3 M 178, procès-verbal d'enquête du 7 septembre 1848.

46. . C'est la conclusion de l'article de Jean-Yves Carluer, « Le gendarme, le maire et les Bas-Bretons : quelques réflexions sur les représentations du pouvoir et la fragilité des épaulettes et des aiguillettes au XIXème siècle ", dans Violence et société en Bretagne et dans les pays celtiques. Actes du colloque international, Brest, 18-20 mars 1999 [réunis par Jean-Yves Carluer], Kreizn ${ }^{\circ}$ 13, Brest, Centre de Recherche Bretonne et CeltiqueUniversité de Bretagne Occidentale, 2000, pp. 505-521.

47. Il conviendrait de superposer à la grande typologie des sociétés rurales (telle qu'elle a été énoncée par Pierre Barral dans : Les agrariens français de Méline à Pisani, Paris, Librairie Armand Colin, 1968 ; la Bretagne appartient, en fonction de ses pays, 
soit aux « démocraties cléricales », soit aux pays de « hiérarchies acceptées », pp. 49-50 et pp. 55-56) une autre typologie fondée sur les conditions d'acceptation ou de refus des normes imposées par un État-superstructure. Aux sociétés de dissidence étudiées, par exemple, par Jean-François Soulet, Christian Thibon et François Ploux, nous pourrions opposer un autre modèle sociétal - celui de la société basse-bretonne - fondé sur une obéissance tactique et/ou volontaire (et non une soumission) à des normes extérieures au monde des communautés villageoises.

48. . Très exceptionnellement, ses décisions sont contestées. A Crozon, le notaire Alavoine présenta une requête devant le Conseil d'État visant à annuler la décision du conseil de préfecture qui avait conclu à la validité des opérations électorales dans la commune. Il fut débouté et son cas est suffisamment rare pour qu'il soit mentionné, A.D.F., 3 M 172, extrait du procès-verbal des délibérations de la section du contentieux, séance du 18 mai 1849.

49. . Ce que propose Olivier Ihl, « Tours de main et double jeu. Les fraudes électorales depuis la Révolution française », op. cit., pp. 77-78.

50. . Archives privées De la Villemarqué de Cornouaille, lettre du 26 août 1848.

51. A.D.F., 3 M 178, avis du 15 septembre 1848.

52. . En juillet 1848 : 305 électeurs inscrits et 202 votants ; 256 votants en octobre : 213 suffrages portés sur chacun des onze premiers conseillers municipaux et 212 sur le douzième, A.D.F., 3 M 178.

53. . « Le système du droit et le champ judiciaire sont le véhicule permanent de rapports de domination, de techniques d'assujettissement polymorphes. Le droit, il faut le voir, je crois, non du côté d'une légitimité à fixer, mais du côté des procédures d'assujettissement qu'il met en oeuvre ", écrit Michel Foucault, "Il faut défendre la société ». Cours au Collège de France. 1976, Paris, Éditions Gallimard-Hautes Études-Le Seuil, 1997, p. 24.

54. . A.D.F., 3 M 179, lettre du 5 août 1848.

55. . "Sur le 2ème grief: considérant que si le nombre des bulletins comptés dans l'urne n'était pas égal au nombre des votants émargés, cette différence n'était d'après les explications plausibles du maire que de cinq à six \& non aussi considérable qu'on le prétend. Considérant que cette différence ne saurait être attribuée à la fraude et qu'elle n'a pu en aucune manière exercer d'influence sur le résultat du scrutin. Considérant que dans les campagnes où se réunissent beaucoup d'hommes illettrés il est presque impossible d'atteindre à la perfection des opérations électorales. Le conseil de préfecture arrête : les élections municipales de la commune de Plonéis sont déclarées valides \& sortiront tout leur effet ", A.D.F., 3 M 179.

\section{INDEX}

Index géographique : France

Mots-clés : démocratie, histoire, surveillance

Index chronologique : 19e siècle 